\section{Melanom bedingt Folgekrebs, Krebs bedingt Folgemelanom}

\author{
Melanome und Krebserkrankungen an anderen Organen sind wechselseitig \\ korreliert. Einer italienischen Studie zufolge liegt das wahrscheinlich an der \\ Art, wie bestimmte Personengruppen leben.
}

$\mathrm{D}$ ie wechselseitigen Assoziationen wurden anhand der Daten von 1.537 Melanompatienten und 52.354 Patienten mit nicht kutanem Krebs errechnet. Hiernach bekamen 76 Melanompatienten ein zweites Primärmalignom, wobei die Diagnose in $64 \%$ der Fälle bereits in den ersten zwei Jahren nach dem Hautkrebsbefund gestellt wurde. Umgekehrt wuchs bei 127 Krebspatienten zusätzlich ein primäres Melanom. Die mediane Nachbeobachtungszeit betrug in beiden Patientengruppen vier Jahre.

Die Forscher kalkulierten aus ihren Daten standardisierte Inzidenzraten
(SIR) als Verhältnis der beobachteten zu den erwarteten Tumorhäufigkeiten.

Bidirektionale Assoziationen wie etwa in Bezug auf Brust-, Schilddrüsenkrebs und hämatopoetische Malignome erklären sich die Autoren einmal mit gemeinsamen, Lebensstil-bedingten Risikofaktoren. Sie weisen darauf hin, dass Melanome häufiger bei Patienten mit höherem sozioökonomischem Status auftreten und sehen darin eine mögliche Verbindung zu Prostata-, Brust- und Schilddrüsenkrebs. Eine andere mögliche Erklärung ist eine systematische Verzerrung, die sich aus den engmaschigen Nachuntersuchungen nach der Diagnose eines Malignoms ergeben kann. Dafür spricht, dass zweite primäre Malignome nach Melanomdiagnose sehr häufig in zwei Jahren nach der Hautkrebsdiagnose diagnostiziert wurden und sich die Melanome, die nach vorheriger nicht kutaner Krebserkrankung gefunden wurden, tendenziell in einem früheren Stadium als erstdiagnostizierte Melanome befanden.

Fazit: Ursachen für Korrelationen zwischen Melanomen und anderen Tumoren sind wahrscheinlich eine soziokulturell bedingte Häufung von Risikofaktoren und eine durch verstärkte Überwachung entstehende Verzerrung bei den gleichen Patientensubgruppen, so die Autoren.

Dr. Robert Bublak

Caini S et al. Risk of second primary malignancies among 1537 melanoma patients and risk of second primary melanoma among 52354 cancer patients in Northern Italy. J Eur Acad Dermatol Venereol. 2016;30:1491-6

\title{
Aktinische Keratose: neue PDT-Strategie mit weniger Nebenwirkungen
}

\begin{abstract}
Viele Patienten mit aktinischer Keratose leiden unter den Nebenwirkungen einer photodynamischen Therapie. Einer dänischen Studie zufolge können diese durch eine veränderte Behandlungsstrategie reduziert werden.
\end{abstract}

$\mathrm{D}$ ie stärksten Nebenwirkungen einer photodynamischen Therapie (PDT) zur Behandlung einer aktinischen Keratose (AK) sind Schmerzen während der Bestrahlung, posttherapeutische Ödeme sowie Hautrötungen und Entzündungserscheinungen.

Im Rahmen einer randomisierten intraindividuellen Studie haben dänische Forscher nach Möglichkeiten gesucht, mit verändertem Prozedere bei gleicher Wirksamkeit weniger Nebeneffekte zu erzielen als mit der konventionellen Methode (PDT mit Methylaminolävulinat, MAL, für drei Stunden). Die Dermatologen verkürzten die Einwirkzeiten des Photosensibilisators auf 30 Minuten (Pulse-PDT) oder behandelten die zu bestrahlenden Hautstellen vor und direkt nach der Pulse-PDT zusätzlich mit einer hochpotenten Kortikoidsalbe (S-Pulse-PDT).
21 immunkompetente Männer und eine Frau mit leichten Formen multipler aktinischer Keratosen im Gesicht und auf der Kopfhaut wurden in jeweils drei ähnlichen Regionen entsprechend unterschiedlich behandelt. Nach drei Stunden wurden alle Regionen der durchschnittlich 76-Jährigen mit Rotlicht bestrahlt. Die Hautrötung wurde in Vorbereitung auf die Bestrahlung sowie am darauffolgenden Tag mit einem Reflektometer bestimmt. AK-Läsionen in allen Therapiebereichen wurden gezählt, eingestuft und dokumentiert. Drei Monate nach der Behandlung wurden die behandelten Gebiete mit dem Ausgangsbefund verglichen.

An den Pulse-PDT-behandelten Stellen ergab sich 24 Stunden nach der Therapie insgesamt eine signifikant schwächere Rötung als nach konventioneller
PDT. Noch weniger Entzündungserscheinungen zeigten sich, wenn zusätzlich Kortisonsalbe appliziert worden war (S-Pulse-PDT). Während sich bei konventionell behandelten Patienten immer eine Rötung ergab, waren bei einem Patienten der Pulse-PDT- und vier Patienten der S-Pulse-PDT-Gruppe keinerlei Erytheme erkennbar. Hellrot mit Verhärtungen zeigten sich die Hauterscheinungen dagegen bei neun Patienten der PDT-Gruppe, bei nur drei der PulsePDT- und bei keinem der S-Pulse-PDTPatienten. Die Behandlungserfolge nach drei Monaten unterschieden sich zwischen den drei Therapiearten nicht signifikant.

Fazit: Eine verkürzte Einwirkzeit des Photosensibilisators und die zusätzliche Applikation lokalen Kortikoids können dazu beitragen, das Nebenwirkungspotenzial der photodynamischen Therapie bei gleicher Effizienz zu reduzieren.

Dr. Christine Starostzik

Wiegell S R et al. Pulse photodynamic therapy reduces inflammation without compromising efficacy in the treatment of multiple mild actinic keratoses of the face and scalp: a randomized clinical trial. Br J Dermatol. 2016;174:979-84 